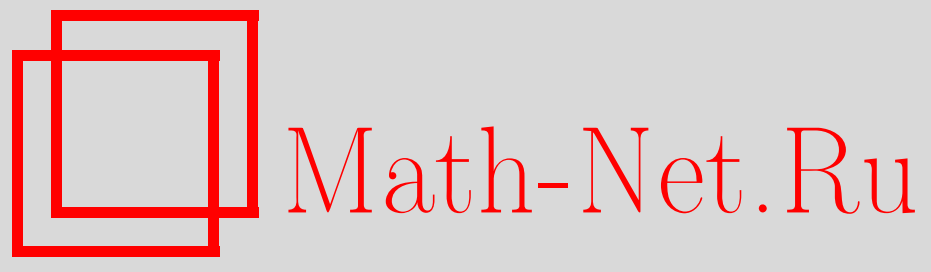

А. И. Овчинников, Порядки дифференцирований в разложении радикальных дифференциальных идеалов, УМH, 2008, том 63, выпуск 2, 179-180

DOI: https://doi.org/10.4213/rm8542

Использование Общероссийского математического портала Math-Net.Ru подразумевает, что вы прочитали и согласны с пользовательским соглашением http://www.mathnet.ru/rus/agreement

Параметры загрузки:

IP : 18.209 .158 .208

26 апреля 2023 г., 16:00:07

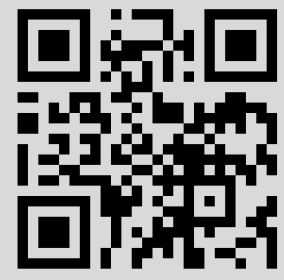




\section{Порядки дифференцирований в разложении радикальных дифференциальных идеалов}

\section{А. И. Овчинников}

1. Введение. Дифференциальное кольцо $R$ - это коммутативное кольцо с единицей и дифференцированиями $\Delta=\left\{\delta_{1}, \ldots, \delta_{m}\right\}$. Случай $\Delta=\{\delta\}$ называется обыкновенным. Под редукцией понимается дифференциальная псевдоредукция [1]-[4]. Дифференциальный и радикальный дифференциальный идеалы, порожденные множеством $F$, обозначают $[F]$ и $\{F\}$ соответственно. Рассматривается кольцо дифференциальных многочленов $\mathbf{k}\left\{y_{1}, \ldots, y_{n}\right\}$. Если $S$ - мультипликативное множество, a $I$ - дифференциальный идеал, то $I: S=\{r \in R \mid s r \in I$ для некоторого $s \in S\}-$ тоже дифференциальный идеал. Предполагаем, что зафиксирован ранжир на производных в кольце $\mathbf{k}\left\{y_{1}, \ldots, y_{n}\right\}$.

ОПРедЕлЕниЕ 1 [1; с. 82]. Авторедуцированное подмножество наименьшего ранга в идеале $I$ называется характеристическим множеством идеала $I$.

Множество инициалов и сепарант авторедуцированного множества $\mathscr{A}$ обозначается через $H_{\mathscr{A}}$, а его мультипликативное замыкание - через $H_{\mathscr{A}}^{\infty}$.

ОпредЕление 2 [5; определение 2.6]. Радикальный дифференциальный идеал $I$ в кольце $\mathbf{k}\left\{y_{1}, \ldots, y_{n}\right\}$ называется характеризуемым, если найдется характеристическое множество $\mathscr{A}$ идеала $I$ такое, что $I=[\mathscr{A}]: H_{\mathscr{A}}^{\infty}$.

Характеризуемость идеала $I$ зависит от ранжира. Однако простые дифференциальные идеалы характеризуемы относительно любого ранжира. Существуют несколько алгоритмов разложения радикального дифференциального идеала в пересечение характеризуемых. Одним из первых был алгоритм Ритта-Колчина. Этот алгоритм разлагал на дифференциальные простые компоненты, используя факторизацию. Мы остановимся на одной из версий алгоритма Rosenfeld-Gröbner [6], [5]. Этот алгоритм не использует факторизацию и реализован в системе компьютерной алгебры Maple.

2. Основной результат. Главная задача - ограничить дифференцирования, производимые при редукции. Этого можно добиться, заменив дифференциальную редукцию на алгебраическую и проведя все дифференцирования заблаговременно. Совместно с О. Голубицким и М. В. Кондратьевой мы доказали, что в случае применения такой специальной редукции, имея систему дифференциальных многочленов $F \subset \mathbf{k}\left\{y_{1}, \ldots, y_{n}\right\}$ на входе, мы получаем на выходе разложение $\{F\}=\left[\mathscr{C}_{1}\right]$ : $H_{\mathscr{C}_{1}}^{\infty} \cap \cdots \cap\left[\mathscr{C}_{k}\right]: H_{\mathscr{C}_{k}}^{\infty}$, которое удовлетворяет следующей оценке:

$$
M\left(\mathscr{C}_{i} \cup H_{\mathscr{C}_{i}}\right) \leqslant(n-1) ! M(F), \quad \text { где } M(F)=\sum_{i=1}^{n} m_{i}(F),
$$

а $m_{i}(F)$ - это максимальный порядок дифференцирований по переменной $y_{i}$ среди элементов $f \in F$.

Хотелось бы иметь оценку для произвольного алгоритма редукции. Если мы редуцируем относительно произвольного дифференциального треугольного множества, результат редукции зависит от выбора пути редукции. Рассмотрим это на примере.

ПримеР 1. Рассмотрим дифференциальное треугольное множество

$$
\mathscr{C}=x(x-1),(x-1) y, z+y+t x
$$

Работа выполнена при поддержке РФФИ (грант № 05-01-00671). 
с исключающим ранжиром $t<x<y<z$ и дифференциальный многочлен $f=$ $z^{\prime}+y^{\prime}$. Мы можем редуцировать $f$ относительно $\mathscr{C}$ многими различными способами, и остатки существенно отличаются:

$$
\begin{aligned}
& z^{\prime}+y^{\prime} \stackrel{z^{\prime}+y^{\prime}+t^{\prime} x+t x^{\prime}}{\longrightarrow} t^{\prime} x+t x^{\prime} \stackrel{(2 x-1) x^{\prime}}{\longrightarrow} t^{\prime}(2 x-1) x=2 t^{\prime} x^{2}-t^{\prime} x \stackrel{x^{2}-x}{\longrightarrow} t^{\prime} x=: f_{1}, \\
& z^{\prime}+y^{\prime} \stackrel{(x-1) y^{\prime}+x^{\prime} y}{\longrightarrow}(x-1) z^{\prime}-x^{\prime} y \stackrel{(x-1) y}{\longrightarrow}(x-1)^{2} z^{\prime} \\
& \stackrel{z^{\prime}+y^{\prime}+t^{\prime} x+t x^{\prime}}{\longrightarrow}(x-1)^{2}\left(y^{\prime}+t^{\prime} x+t x^{\prime}\right) \longrightarrow 0=: f_{2} .
\end{aligned}
$$

Мы видим, что остаток $f_{1}$ зависит от переменной $t^{\prime}$, которой нет ни в $f_{2}$, ни в $\mathscr{C}$. Так что наряду с остатком, содержащим нетривиальную производную по $t$, находится другой остаток, ее не содержащий.

Основываясь на упомянутом выше результате и идее, продемонстрированной в примере 1 , можно доказать следующую теорему. (Упомянутая в теореме процедуpa Truncate берет многочлен $f$ и удаляет из него все мономы, которые превышают заданную оценку $p_{i}$ по переменной $y_{i}$.)

Теорема 1. Пусть $\mathscr{C}$ - когерентное дифференциальное треугольное множество (см. [7]) дифференииальных многочленов и $f$ - дифференциальный многочлен. Пусть также $p_{i} \geqslant m_{i}(\mathscr{C}), i=1, \ldots, n$. Предположим, что найдется дифференциальный остаток $f$ относительно $\mathscr{C}$, который не содержит производных по дифференциальной переменной $y_{i}$ порядка выше чем $p_{i}, i=1, \ldots, n$. Пусть $g$ - любой дифференииальный остаток $f$ относительно $\mathscr{C}$. Тогда Truncate $\left(g,\left\{p_{i}\right\}\right)$ - дифференииальный остаток $f$ относительно $\mathscr{C}$, в котором порядок по каждой дифференииальной неизвестной $y_{i}$ не превосходит $p_{i}$.

Таким образом, мы получаем оценку (1) для обычного алгоритма RosenfeldGröbner, в котором после проведения каждой дифференциальной редукции нужно выполнять процедуру Truncate.

ЗАмечАниЕ 1 . В заключение заметим, что оценка (1) имеет множитель $(n-1)$ !, где $n$ - число дифференциальных переменных. Он возникает в ходе получения оценки алгоритма "алгебраической" редукции Differentiate \& Autoreduce, эмулирующей дифференциальную. Остается неизвестным, является ли этот множитель $(n-1)$ ! необходимым. Приведем пример, в котором появляется множитель $n-1$.

ПримеР 2. Начнем с системы $F=x_{1}^{\prime}, x_{1}+\cdots+x_{n}$ и исключающего ранжира $x_{1}>\cdots>x_{n}$. Заметим, что $M(F)=1$. После проведения первой редукции имеем: $F_{\text {new }}=x_{2}^{\prime}+\cdots+x_{n}^{\prime}, x_{1}+\cdots+x_{n}$. Так что $M\left(F_{\text {new }}\right)=n-1=(n-1) M(F)$.

Автор благодарен Е.В. Панкратьеву, М.В. Кондратьевой и О. Голубицкому за важные комментарии и поддержку, и А. Зобнину за важные замечания и предложения.

\section{Список литературы}

[1] E. R. Kolchin, Differential algebra and algebraic groups, Pure Appl. Math., 54, Academic Press, New York-London, 1973. [2] M. V. Kondratieva, A. B. Levin, A. Mikhalev, E. V. Pankratiev, Differential and difference dimension polynomials, Math. Appl., 461, Kluwer, Dordrecht, 1999. [3] J. F. Ritt, Differential algebra, Amer. Math. Soc. Colloq. Publ., 33, Amer. Math. Soc., New York, 1950. [4] W. Sit, Proc. Amer. Math. Soc., 68:3 (1978), 251-257. [5] E. Hubert, J. Symbolic Comput., 29:4-5 (2000), 641-662. [6] F. Boulier, D. Lazard, F. Ollivier, M. Petitot, Proceedings of ISSAC (Montreal, 1995), ACM Press, New York, 1995, 158-166. [7] E. Hubert, Lecture Notes in Comput. Sci., 2630 (2003), 40-87.

А. И. Овчинников (А. I. Ovchinnikov)

Московский государственный университет им. М. В. Ломоносова

E-mail: alexey_ov@yahoo.com
Представлено А. В. Михалёвым Принято редколлегией 09.12.2007 